\title{
Emotion Regulation Strategies and Stress in Irish College Students and Chinese International College Students in Ireland
}

\author{
Yuning Sun \\ Conor Nolan \\ National College of Ireland, Ireland
}

\begin{abstract}
Little is known about the association between emotion regulation strategies and perceived stress in college students, and in particular, the strategies used by international students. The present research examined if differences exist in the use of emotion regulation strategies between Irish college students and Chinese international college students studying in Ireland, and investigated the relationship between emotion regulation strategies and perceived stress in these two student populations. Chinese students reported more frequent use of cognitive reappraisal and expressive suppression compared with Irish students. There was a statistically significant negative association between the habitual use of cognitive reappraisal and levels of stress in both Irish and Chinese students. There was a significant positive relationship between the habitual use of expressive suppression and levels of stress in Irish college students, but not in Chinese students. The findings highlight the effects of emotion regulation on levels of stress and the importance of cultural context when investigating the association between emotion regulation strategies and perceived stress in students.
\end{abstract}

Keywords: Chinese, college students, emotion regulation, Irish, stress 
According to the Economic and Social Research Institute, the number of international students coming to Ireland continues to increase year to year, with an increase of $45 \%$ between the years 2013 and 2017. During these years, China was the top country of origin for non-European students studying in Ireland (see Groarke \& Durst, 2019 for full details and figures). This could be due, in part, to the fact that Irish higher education institutes (HEIs) have directly targeted China in their marketing strategies (Clarke et al., 2018). A number of Irish HEIs have partnerships with, and campuses located in, China (Groarke \& Durst, 2019) with many of them providing opportunities for Chinese students to study in Ireland. This suggests that there is a considerable degree of student mobility between China and Ireland and supports the rationale for focusing on Chinese international students in Ireland in the present research.

When considering the differences between Ireland and China, Ireland is considered as an individualistic culture, where independence and the autonomy of self is strongly emphasized. However, China is considered as a collectivistic culture, where group relations and group harmony are greatly emphasized (Hofstede, 2001). Hence, it may be particularly stressful for Chinese international students to adjust to the new cultural environment (Chen et al., 2020; Leong, 2015). Additionally, research has indicated that international college students may experience stress due to insufficient language skills, a lack of friends, and homesickness (Forbush \& Foucault-Welles, 2016; Harrer et al., 2018).

There are a variety of strategies that international students may use to deal with such stressors and to integrate into the host country (Sullivan \& KashubeckWest, 2015). For example, research on international Chinese students has indicated that socializing and making friends can help these students deal with stressors in their new host country environment (Cheng et al., 2019). A further variable of interest in relation to stress and international students is the use of emotion regulation strategies. It is well outlined in the existing literature that the ability to manage one's emotions in an adaptive manner is critical to mental health (Chervonsky \& Hunt, 2019). However, cross-cultural studies have found that people from different cultures may have different ways of understanding, expressing, and coping with emotions (Yoo et al., 2006). For example, in individualistic cultures (such as Ireland), expressive suppression could compromise individuality. In collectivistic cultures (such as China), open emotional expression may be incongruent with their social value systems and cause a disruption to group harmony and group relations (Butler et al., 2007). This suggests that emotion regulation strategies that are typical in the country of origin for international students may differ significantly from those commonly seen in the host country.

There is an absence of research that considers the relationship between emotion regulation (hereafter ER) and stress management among international students. Furthermore, relatively little is known about the difference in ER and stress management between local and international college students. There have been a large number of studies showing that international college students face difficulties in adapting to host cultures and usually experience higher levels of stress when studying in an unfamiliar culture (Cho \& Yu, 2015; Han et al., 2017); 
therefore, it would be of value to examine which ER strategies are beneficial for this population. In addition, as third-level education can be a stressful experience, colleges may want to introduce emotion management workshops for students, particularly on how to reappraise negative thoughts and stressful events, to help international students adapt to college life and reduce levels of stress.

The current study focuses on Chinese international college students in the host country (Ireland) and investigates how ER influences perceived stress among Chinese international students, and compares them to their host country peers. The first aim of the current research is to examine if differences exist in the habitual use of ER strategies between Irish college students and Chinese international students studying in Ireland. The second aim is to investigate the relationship between ER strategies and perceived stress in these two student populations.

\section{LITERATURE REVIEW}

ER can be defined as "extrinsic and intrinsic processes responsible for monitoring, evaluating, and modifying emotional reactions, especially their intensive and temporal features, to accomplish one's goals" (Thompson, 1994, p. 27). As described by Thompson (1994), the intensive features relate to the intensity of the experienced emotion, and the temporal features relate to onset or persistence of the emotion felt. ER has been found to be broadly related to psychosocial adjustment and mental health (Zahniser \& Conley, 2018). Although there are many different frameworks that help to conceptualize the diverse ways individuals can regulate their emotions, the process model of ER has received empirical support from numerous studies and has been widely used to illustrate ER processes (Gross, 1998a, 1998b, 2001, 2013, 2015).

According to the process model, emotions tend to be regulated by numerous processes that involve specific strategies. Among those strategies, cognitive reappraisal and expressive suppression have been identified as commonly used and valuable strategies and have received a considerable amount of attention in the literature (Gresham \& Gullone, 2012).

\section{Cognitive Reappraisal, Expressive Suppression, and Mental Health}

Cognitive reappraisal involves reinterpreting emotion-eliciting situations in order to change or modulate emotional meaning (Troy et al., 2010). For example, individuals may reinterpret an insult as being reflective of the character of the insulter rather than their own character. That is, by using cognitive reappraisal, individuals attempt to reinterpret an emotion-eliciting situation in a way that alters its meaning and changes its emotional impact. In addition, reappraisal intervenes relatively early and can occur before emotions have been fully elicited and thus may alter the entire subsequent emotional trajectory (Goldin et al., 2008). The habitual use of cognitive reappraisal is usually considered as an adaptive strategy and has been found to be linked with many desirable outcomes, such as positive 
emotions, closer interpersonal relationships, a high level of willingness to seek social support, and enhanced well-being (English et al., 2012).

However, Gross (2015) noted that expressive suppression involves a continuous focus on the self in order to prevent the activation of emotional responses. For example, by using expressive suppression, people may hide an emotional response when they hear bad news or may remain silent when they hear something offensive. Existing research has indicated that individuals who use expressive suppression to manage negative emotions, such as anxiety or sadness, are more likely to experience a high intensity of those negative emotions, whereas individuals who use expressive suppression to manage positive emotions, such as happiness, are more likely to dampen their positive emotional experiences (Campbell-Sills et al., 2006). It should be noted that the research discussed above primarily focused on Western populations. Butler and Gross (2009) outlined that it is necessary to consider cultural values when investigating the effects of emotional expression on mental health, particularly when considering other populations.

\section{ER Strategies and Stress}

When specifically considering how ER strategies relate to stress responses and stressors, research again suggests that cognitive reappraisal and expressive suppression play key roles; however, the majority of research in this area has examined clinical levels of stress and psychopathologies such as post-traumatic stress disorder. Moore and colleagues (2008) investigated the relationship between cognitive reappraisal and expressive suppression and measures of stressrelated reactions in a sample of undergraduate students and trauma-exposed individuals. Their research suggested that expressive suppression was associated with higher self-reported stress symptoms and reappraisal with lower selfreported stress symptoms. Their study did not include a direct measure of stress, but showed the possibility that the habitual use of either cognitive reappraisal or expressive suppression may influence levels of stress. It is also worth noting that the sample was an American sample, with predominantly Caucasian respondents.

Troy and colleagues (2010) found that the habitual use of cognitive reappraisal was associated with fewer depressive symptoms among female participants who reported a higher level of cumulative life stress. The authors concluded that cognitive reappraisal could be regarded as a necessary buffer for individuals who experience higher levels of cumulative life stress. Longitudinal research has also investigated cognitive reappraisal strategies in stressed populations, such as people living with HIV and careers of people with cystic fibrosis (Carrico et al., 2005; Pakenham, 2005). Both of these pieces of research supported the adaptive effects of cognitive reappraisal. However, Troy and Mauss (2011) highlighted that the efficacy of cognitive reappraisal is not well understood, and that much of the existing research focuses on one specific stressor, making it difficult to generalize results across types and intensities of stressors. 
Few studies have investigated the relationship between ER strategies and levels of stress in student and international student populations. One recent piece of research did investigate this topic, using qualitative methods. Cheng and colleagues (2019) investigated how Chinese students in the United Kingdom coped with challenges by using ER strategies. From their interviews they identified that their sample of international Chinese students used strategies that aligned with Gross' (1998a) categorization of ER strategies, including expressive suppression and cognitive reappraisal. It is of value to further investigate the use of ER strategies in a larger sample of international Chinese students, and whether these differ from the strategies used by students in the host country. In addition, the ability to use ER strategies is considered an important skill during college life, as most college students are in the period of emerging adulthood (Zahniser \& Conley, 2018). This period may be accompanied by pressures from both academics and daily life, such as academic pressures, relationships with friends and family, and financial constraints (Rankin et al., 2018). Therefore, there is value in understanding this for mental health, stress, and support reasons among both local and international students.

\section{Theoretical Framework}

Fundamentally, culture is commonly conceptualized as shared symbols, norms, and values specific to a group of people. The most influential cultural theory is Hofstede's national cultural framework (Hofstede, 2001). Within the framework, individualism-collectivism is typically regarded as the most important dimension (Oyserman \& Lee, 2008). For example, many cross-cultural psychological differences, such as ER, attitudes, and cognitive styles, have been demonstrated as a dichotomy between a collectivistic orientation found in Asian countries and an individualistic orientation found in Western countries (Minkov et al., 2017). Specifically, people from individualistic cultures emphasize their independence of others, and focus more on personal goals, desires, and motivations (Mesquita, 2001). People within collectivistic cultures generally describe themselves in ways that emphasize empathy and their relatedness to others (Singelis et al., 1995).

Previous research has found that there is a difference in the habitual use of ER strategies and their effects between individualistic and collectivistic cultures (Butler et al., 2007). For instance, people from collectivistic cultures are more likely to report less emotion during an elicitation task and are more likely to report the frequent use of expressive suppression (Mauss \& Butler, 2010). One possible explanation could be that free and open emotional expression, particularly of negative emotions, may cause disruptions in group harmony, which has always been highly valued in collectivistic cultures, where any disruptions may be considered as unacceptable (Soto et al., 2011). In individualistic cultures, group harmony has less relevance and the free expression of emotions is more often emphasized (Frijda \& Sundararajan, 2007). Moreover, Soto and colleagues (2011) reported that the habitual use of cognitive reappraisal was correlated with positive 
mental health outcomes, such as higher levels of life satisfaction and lower levels of anxiety or depression in both Asian and European-American participants.

Yet, the habitual use of expressive suppression may have different consequences for different cultural groups. For example, more expressive suppression is related to negative mental health outcomes, such as higher levels of anxiety and lower levels of positive affect, only among individuals from an individualistic culture (e.g., European or American). In contrast, more expressive suppression is associated with positive outcomes among those from a collectivistic culture (e.g., Asian), such as lower levels of loneliness and improved emotional recovery (Su et al., 2013). Research by Wei and colleagues (2013) suggested that Asian students tend to regulate emotions on their own. The reason for this may be due to perceived shame or embarrassment associated with seeking social support. This is also discussed by Smith and Khawaja (2011) as reasoning for why Asian students may engage in higher levels of suppression as a form of emotion control.

Therefore, based on the culture value dimension of individualismcollectivism and previous research on cultural differences in ER, the following hypotheses were formulated.

Hypothesis 1: Chinese international college students will report more frequent use of expressive suppression than Irish college students.

Hypothesis 2: There will be no difference in the habitual use of cognitive reappraisal between Irish and Chinese students.

Hypothesis 3: There will be a negative relationship between habitual use of cognitive reappraisal and perceived stress in Irish students.

Hypothesis 4: There will be a negative relationship between habitual use of cognitive reappraisal and perceived stress in Chinese students.

Hypothesis 5: Nationality moderates the association between the habitual use of expressive suppression and perceived stress. Specifically, the association between the more frequent use of expressive suppression and higher levels of stress will be seen in Irish students but not in Chinese students.

\section{METHOD}

\section{Participants}

Inclusion criteria required participants to be over 18 years old, currently studying in a college or university in Ireland (undergraduate or postgraduate), and to self-identify as an Irish or international Chinese student. All data were collected online using convenience sampling. Specifically, we recruited participants through social networks, such as Facebook, and student WeChat groups (similar to WhatsApp groups), which the first author had access to. We also invited participants to share the survey link with other individuals who satisfied the 
inclusion criteria (Noy, 2008). International offices in five higher education institutions in Ireland posted the survey link on their social media sites.

Table 1 outlines frequencies for all demographic variables. A total of 170 college students participated in the current study. They were aged between 18 and 36 , with a mean age of 23.43 years $(S D=3.28)$. The 170 participants consisted of 93 undergraduate and 77 postgraduate students. With respect to Irish college students, 74 participants (44 females, 30 males; $M=22.84$ years, $S D=2.94$ ) completed the online questionnaire. All the participants in this group selfidentified as Irish and were currently studying in a college or university in Ireland. With respect to Chinese international college students, 96 participants (56 females, 40 males; $M=23.89$ years, $S D=3.47$ ) completed the online questionnaire. All the participants in this group self-identified as Chinese and were international students in one of the universities or colleges in Ireland. The majority of Chinese international students $(n=46)$ had spent $1-3$ years in Ireland at the time of data collection, 22 Chinese participants had spent less than 1 year, and 28 Chinese participants had spent more than 3 years in Ireland.

Table 1: Frequencies and Valid Percentages for All Demographic Variables

\begin{tabular}{lllll}
\hline Variable & \multicolumn{2}{l}{ Frequency $(n)$} & \multicolumn{2}{c}{ Valid percentage (\%) } \\
\cline { 2 - 5 } & $\begin{array}{l}\text { Chinese } \\
\text { students }\end{array}$ & $\begin{array}{l}\text { Irish } \\
\text { students }\end{array}$ & $\begin{array}{l}\text { Chinese } \\
\text { students }\end{array}$ & $\begin{array}{l}\text { Irish } \\
\text { students }\end{array}$ \\
\hline Gender & & & & \\
$\quad$ Male & 40 & 30 & 41.7 & 40.5 \\
$\quad$ Female & 56 & 44 & 58.3 & 59.5 \\
Age & & & & \\
$\quad$ 18-25 & 67 & 61 & 69.8 & 82.4 \\
$26-30$ & 27 & 10 & 28.1 & 13.5 \\
$\quad 31+$ & 2 & 3 & 2.1 & 4.1 \\
$\begin{array}{l}\text { Degree program } \\
\text { Undergraduate }\end{array}$ & 45 & 48 & 46.9 & 64.9 \\
$\quad \begin{array}{l}\text { Postgraduate } \\
\text { Length of stay in Ireland }\end{array}$ & 26 & 53.1 & 35.1 \\
$\quad$ Less than & & & & \\
1 year & 22 & & 22.9 & \\
1-3 years & 46 & & 47.9 & \\
$\quad \begin{array}{l}\text { More than } \\
\text { 3 years }\end{array}$ & 28 & & 29.2 & \\
\hline
\end{tabular}

\section{Measures}

Demographic variables in the current study consist of gender, age, nationality, degree program, and length of stay in Ireland (the latter for Chinese international college students only). The two psychological measures used in the current study were the Emotion Regulation Questionnaire (ERQ; Gross \& John, 2003), and the Perceived Stress Scale (PSS; Cohen et al., 1983). 
The ERQ was used to assess ER strategies. The 10-item scale, which is rated on a 7-point Likert scale ( 1 = strongly disagree, $7=$ strongly agree $)$, includes two dimensions that assess subjective habitual use of cognitive reappraisal and expressive suppression. We computed a total score for each subscale by adding up the responses to each relevant item. Higher scores on the subscales indicate more frequent use of the ER strategy. The ERQ has been found to have good construct validity, adequate internal consistency for both cognitive reappraisal $(\alpha=.79)$, and expressive suppression subscales $(\alpha=.73)$, and adequate test-retest reliability ( $r=.69$; Gross \& John, 2003). In the current study, Cronbach's alphas were .94 and .86 for cognitive reappraisal and expressive suppression, respectively, in the Irish group. Cronbach's alphas were .81 and .69 for cognitive reappraisal and expressive suppression, respectively, in the Chinese group.

The PSS was used to measure the degree to which life circumstances are appraised as stressful. The 10-item survey was rated on a 5-point Likert scale $(0=$ never, $4=$ very often $)$. Total scores can range from 0 to 40 , with higher scores indicating a greater degree of perceived stress. We computed the total by adding up the responses to each item. The scale has been widely used for assessing nonspecific perceived stress due to ease of administration and it also has been found to have adequate 2-day $(r=.85)$ and 6-week $(r=.55)$ test-retest reliability, and good convergent validity (Cohen et al., 1983; Myers et al., 2012). In the current study, Cronbach's alphas were .93 for the Irish group and .83 for the Chinese group.

\section{Procedure}

Once participants clicked the relevant link they were directed to a host website that provided them with an information and consent page. Once participants indicated their consent and that they had read the information page, they were directed to the demographic questionnaire, followed by the ERQ and the PSS. After completing the required questionnaires, participants were presented with a debriefing page. The data were collected from February 2019 to March 2019.

\section{Data Analysis}

We carried out descriptive analyses for all the key variables, including means, standard deviations, and assumption testing. Due to sample size and nonnormal distribution, we used the Mann-Whitney $U$ test to determine if there were differences in cognitive reappraisal and expressive suppression scores between Irish students and Chinese students. We conducted a hierarchical regression analysis to investigate the relationship between cognitive reappraisal, expressive suppression, and perceived stress by nationality. We included demographic control variables in the regression. A hierarchical regression is appropriate here as it allows for the investigation of the relationship between these variables, while considering an interaction term (interaction between ER strategy and nationality) and demographic control variables. 


\section{RESULTS}

Table 2 outlines the average scores for Irish and Chinese participants on the cognitive appraisal, expressive suppression, and perceived stressed measures.

Table 2: Descriptive Statistics of All Continuous Variables

\begin{tabular}{lcccccc}
\hline Variable & $M$ & $95 \%$ CI & SEM & Median & SD & Range \\
\hline Cognitive reappraisal & & & & & & \\
$\quad$ Chinese students & 29.05 & {$[27.94,30.22]$} & .56 & 29.0 & 5.49 & $15-42$ \\
$\quad$ Irish students & 24.26 & {$[22.41,26.08]$} & .95 & 22.5 & 8.21 & $12-41$ \\
$\begin{array}{l}\text { Expressive suppression } \\
\text { Chinese students }\end{array}$ & 16.45 & {$[15.47,17.34]$} & .49 & 16.5 & 4.84 & $4-28$ \\
$\quad \begin{array}{l}\text { Irish students } \\
\text { Perceived stress }\end{array}$ & 9.46 & {$[8.58,10.41]$} & .46 & 9.0 & 4.00 & $4-26$ \\
$\quad$ Chinese students & 20.95 & {$[19.90,21.95]$} & .54 & 21.00 & 5.32 & $9-40$ \\
$\quad$ Irish students & 23.43 & {$[21.6,25.2]$} & .91 & 23.00 & 7.89 & $8-36$ \\
\hline
\end{tabular}

\section{Differences in Expressive Suppression and Cognitive Reappraisal Scores}

A Mann-Whitney $U$ test indicated that cognitive reappraisal scores for Chinese students (median $=29$, mean rank $=98.69$ ) were statistically significantly higher than for Irish students (median $=22.5$, mean rank $=68.51 ; U=4,809, z=$ $3.95, p<.001)$. The effect size was medium $(r=.3)$, according to Cohen $(1988)$.

Expressive suppression scores for Chinese students (median $=16.5$, mean rank $=113.26)$ were statistically significantly higher than for Irish students (median $=9$, mean rank $=49.49 ; U=6216, z=8.39, p<.001$ ). The effect size was large $(r=.6)$, according to Cohen (1988).

\section{Relationship Between Cognitive Reappraisal, Expressive Suppression, and Perceived Stress}

We performed a hierarchical multiple regression analysis to investigate if cognitive reappraisal could predict perceived stress, after controlling for demographic variables. In the first step, three variables were included: gender, age, degree program (undergraduate or postgraduate). This model was not statistically significant: $F(3,165)=1.70, p=.17$. In the second step, two variables were included: nationality and cognitive reappraisal scores. This model was statistically significant $-F(5,163)=22.27, p<.001$-and explained $38.8 \%$ of variance in levels of stress. After the entry of interaction term (Cognitive 
Reappraisal $\times$ Nationality) at Step 3, the total variance explained by the model was $43.1 \%(F[6,162]=22.20 ; p<.001)$. There was a statistically significant moderator effect of nationality, as evidenced by a statistically significant increase in total variation explained of $4.5 \%(F[1,162]=13.38, p<.001$; see Table 3$)$. Simple slopes analysis revealed that there was a statistically significant negative linear relationship between habitual use of cognitive reappraisal and levels of perceived stress in both Irish college students $(B=-.73, S E=.07), p<.001$, and in Chinese international college students $(B=-.29, S E=.10), p=.002$. However, the strength of the relationship is stronger in Irish college students than in Chinese international college students.

Table 3: Hierarchical Multiple Regression Model Predicting Levels of Stress

\begin{tabular}{llllrlrr}
\hline Variable & $R$ & $R^{2}$ & $R^{2}$ change & $B$ & $S E$ & $\beta$ & \multicolumn{1}{c}{$t$} \\
\hline Step 1 & .17 & .03 & & & & & \\
Gender & & & & 0.02 & 1.04 & .001 & 0.02 \\
Age & & & & -0.21 & 0.22 & -.10 & -0.96 \\
Degree & & & & -1.13 & 1.44 & -.08 & -0.79 \\
program & & & & & & & \\
Step 2 & .64 & $.41^{* * *}$ & $.38^{* * *}$ & & & & \\
CR & & & & -0.58 & 0.60 & $-.63^{* * *}$ & -9.81 \\
Nationality & & & & 0.42 & 0.87 & .03 & 0.49 \\
Step 3 & .67 & $.45^{* * *}$ & $.04^{* * *}$ & & & & \\
CR & & & & -0.73 & 0.07 & $.80^{* * *}$ & -10.38 \\
Nationality & & & & -11.42 & 3.34 & $-.85^{*}$ & -3.41 \\
CR x & & & & & & & \\
Nationality & & & & 0.43 & 0.12 & $.97^{* * *}$ & 3.66 \\
\hline
\end{tabular}

Note: $\mathrm{CR}=$ cognitive reappraisal.

${ }^{*} p<.05 ;{ }^{* * *} p<.001$.

We performed a hierarchical multiple regression analysis to investigate if expressive suppression could predict perceived stress, after controlling for demographic variables.

In the first step, we included three variables: gender, age, and degree program (undergraduate or postgraduate). This model was not statistically significant: $F(3$, $165)=1.69, p=.16$. In the second step, two variables were included: nationality and expressive suppression scores. This model was statistically significant $-F(5$, $163)=3.37, p=.006$ - and explained $9.4 \%$ of variance in levels of stress. After the entry of interaction term (Expressive Suppression $\times$ Nationality) at Step 3, the total variance explained by the model was $18.4 \%(F[6,162]=6.08 ; p<.001)$. There was a statistically significant moderator effect of nationality, as evidenced by a statistically significant increase in total variation explained of $9 \%, F(1,162)=$ $17.90, p<.001$ (see Table 4). Simple slopes analysis revealed that there was a statistically significant positive linear relationship $(B=.91, S E=.18)$ between 
habitual use of expressive suppression and levels of perceived stress in Irish college students, $p<.001$, but not in Chinese international college students $(B=-.04, S E=.13 ; p=.78)$.

Table 4: Hierarchical Multiple Regression Model Predicting Levels of Stress

\begin{tabular}{llllrllc}
\hline & $R$ & $R^{2}$ & $R^{2}$ change & $B$ & $S E$ & $\beta$ & \multicolumn{1}{c}{$t$} \\
\hline Step 1 & .17 & .03 & & & & & \\
Gender & & & & 0.02 & 1.04 & .001 & 0.02 \\
Age & & & & -0.21 & 0.22 & -.10 & -0.96 \\
Degree & & & & -1.13 & 1.44 & -.08 & -0.79 \\
program & & & & & & & \\
Step 2 & .31 & $.09^{*}$ & $.06^{*}$ & & & & \\
ES & & & & 0.30 & 0.11 & $.25^{*}$ & 2.63 \\
Nationality & & & & -4.24 & 1.29 & $-.32^{*}$ & -3.29 \\
Step 3 & .43 & $.18^{* * *}$ & $.09^{* * *}$ & & & & - \\
ES & & & & 0.91 & 0.18 & $.77^{* * *}$ & 5.05 \\
Nationality & & & & 7.06 & 2.94 & $.53^{*}$ & 2.40 \\
ES & & & & -0.95 & 0.22 & $-.13^{* * *}$ & -4.23 \\
Nationality & & & & & & & \\
\hline
\end{tabular}

Note: $\mathrm{ES}=$ Expressive Suppression

${ }^{*} p<.05 ;{ }^{* * *} p<.001$.

\section{DISCUSSION}

The aim of the current research was to investigate whether there is a difference in the habitual use of ER strategies (cognitive reappraisal and expressive suppression) between Irish college students and Chinese international college students, and to examine the association between these two ER strategies and levels of stress in Irish and Chinese students. The current research contributes to the understanding of how international students, in this case specifically Chinese students, may deal with stressors in their host country and how these responses may differ to their Western peers. Furthermore, this research also contributes to the field of ER and mental health by investigating the relationship between ER strategies and perceived stress among college students and international students.

\section{Expressive Suppression and Perceived Stress}

Results found that expressive suppression scores for Chinese students were statistically higher than for Irish students, indicating that Chinese students reported more frequent use of expressive suppression than Irish students. This finding is in line with previous research such as that by Soto and colleagues (2011), where Chinese college students reported using expressive suppression with significantly greater frequency than European American college students. Matsumoto and colleagues (2008) demonstrated that participants from Western 
countries, such as the United States and Canada, reported less use of expressive suppression than participants from East Asian countries, such as China, Japan, and Korea.

The current finding is also congruent with the culture norms of emotion suppression. For example, expressive suppression is encouraged and used more frequently in East Asian cultures (Sun \& Lau, 2018). People from East Asian cultures (i.e., collectivistic cultures) are more likely to define themselves based on their social roles and relationships with social groups and are more likely to value the needs of their groups over their personal needs. Hence, expressive suppression is used more frequently in that culture, as it is helpful for achieving group goals, preserving relationships with social groups, and maintaining social harmony (Tsai \& Lu, 2018). In contrast, expressive suppression is discouraged in Western cultures (Roberts et al., 2008). From an early age, children from Western countries (i.e., individualistic cultures) are encouraged to speak up and express their identity and needs through emotional expression (Matsumoto, 2006). Furthermore, emotional expression is considered as a way of asserting individuality and autonomy, which could also be regarded as a societal norm in European American cultural contexts (Su et al., 2015). This societal norm may result in less frequent use of expressive suppression among Irish college students compared with the international Chinese students in the current study.

The current research also found a statistically significant moderator effect of nationality. That is to say, the association between the use of expressive suppression and levels of stress was different for Irish students and Chinese students. Specifically, there was a significant positive relationship between the habitual use of expressive suppression and levels of stress in Irish students, whereas this relationship was absent in Chinese students. Again, this is congruent with previous findings. For instance, Soto and colleagues (2011) found that expressive suppression was correlated with adverse psychological functioning for European American students, but not for Chinese students. Butler and colleagues (2007) reported that the habitual use of expressive suppression was linked with negative emotional experience in participants who hold Western-European values, but this relationship was reversed in participants who hold Asian values. In their meta-analysis, Hu and colleagues (2014) also reported that the effect of expressive suppression on negative mental health outcomes differs between Western and Eastern cultures, as the strength of the association between the frequent use of expressive suppression and negative mental health outcomes was stronger for Western participants.

The current results on the effect of nationality are also consistent with the idea that culture may shape adaptive or maladaptive ER (Wei et al., 2013). As mentioned previously, expressive suppression is generally regarded as maladaptive in individualistic cultural contexts (e.g., Ireland), as it may discourage individualistically focused behaviors such as authenticity and selfassertion. However, the habitual use of expressive suppression could be more valuable in collectivistic cultural contexts (e.g., China), as it could be used to achieve goals and group harmony (Mauss et al., 2010). Therefore, behaviors that are congruent with a culture's values may receive more social reward and become 
more practiced (Ford \& Mauss, 2015). As a result, the habitual use of expressive suppression in Irish college students is not congruent with the cultural context and hence associated with higher levels of stress in the current study. In contrast, this relationship was absent in Chinese students, as the habitual use of expressive suppression is consistent with Chinese cultural context.

Indeed, research has indicated that emotions are actually regulated more quickly through suppression than reappraisal for Chinese individuals (Yuan et al., 2014). Similar findings are suggested from the current research and can add to the argument that suppression is an effective stress strategy for Chinese students. This should be considered in a practical sense. As previously outlined, suppression may not be viewed as culturally beneficial in an Irish context, and may be seen as maladaptive. It may be beneficial for student supports provided by Irish HEIs to help Chinese students to differentiate ER strategies in different contexts and increase their flexibility with respect to when and with whom to suppress their emotions (Wei et al., 2013).

\section{Cognitive Reappraisal and Perceived Stress}

Cognitive reappraisal scores for Chinese students were statistically significantly higher than for Irish students, indicating that Chinese students reported use of cognitive reappraisal more frequently compared with Irish students. Interestingly this contradicts the research by Soto and colleagues (2011) who reported that there were no statistically significantly differences in the habitual use of cognitive reappraisal between Chinese and European American students. Kwon and colleagues (2013) also found there were no cultural differences (i.e., Western cultures and Eastern Asian cultures) in the habitual use of cognitive reappraisal between Korean and American college students.

There was a statistically significant negative association between the habitual use of cognitive reappraisal and levels of stress in both Irish and Chinese students, indicating that the more frequent use of cognitive reappraisal, the lower levels of stress participants may experience. These results are congruent with previous studies. For example, more frequent use of cognitive reappraisal has been found to be correlated with less perceived stress in various population samples such as psychology graduate students (Myers et al., 2012) and adolescents (Boyes et al., 2016). Furthermore, research has indicated that increased use of cognitive reappraisal can also be negatively related to both behavioral and emotional problems (Flouri \& Mavroveli, 2013) and can be positively related to positive mental health outcomes (Hu et al., 2014). However, it is worth noting that the strength of the relationship between the habitual use of cognitive reappraisal and lower levels of stress is weaker for Chinese students than for Irish students in the current study. Although some studies have reported that there is no significant moderating effect of culture or nationality on the association between the use of cognitive reappraisal and mental health, the current study found that nationality moderates the association between the use of cognitive reappraisal and perceived stress. It is still necessary for future research to investigate whether the role of 
culture influences the strength of that association among the general population (Potthoff et al., 2016).

\section{Limitations}

There are some limitations that should be considered in relation to the current research. The sample size and imbalance in nationality numbers should be considered when reviewing the results of the current study. Future research, using larger sample sizes, may also wish to consider the benefits of applying structural equation modeling procedures to their data (Barrett, 2007; Westland, 2012).

It should also be noted that all participants completed the original English version of the questionnaires. There is potential that this could have impacted Chinese students' understanding of the questionnaires. However, the minimum language requirement for international students entering third-level institutions in Ireland is upper intermediate level, meaning that participants have the ability to understand questionnaires and give responses (Sawir et al., 2008).

\section{Future Research}

It would be of benefit for future research to use different methods to measure ER and to investigate if the results of the current study could be replicated. For example, future research could assess daily ER strategies used by participants and examine how the effects of ER on levels of stress vary on a daily basis (Blalock et al., 2016), and investigate individual characteristics that contribute to different ER strategies. The design of the present study means that results are correlational and should be interpreted as such. It may be of benefit for future research to investigate causal relationships between the variables. Future research may also wish to investigate the relationship between ER strategies and more specific stressors (e.g., academic stress). It would also be of value for future research to investigate whether the length of stay among international students in the host country influences the ER or influences the association between ER strategies and levels of stress. It should be noted that the current study did not find significant differences in ER strategies among Chinese international students who spent less than 1 year, 1-3 years, and more than 3 years at the time of data collection in Ireland. Future research may wish to consider length of stay as a continuous variable and the effect of such on ER strategies, as researchers have found individuals who interact or cooperate with local people over time are more likely to have similar patterns of emotional experience (De Leersnyder et al., 2011). In addition, qualitative approaches may provide rich and detailed information about delivery of mental health services (Palinkas, 2014; Xiong \& Zhou, 2018). Future research, involving mixed qualitative and quantitative methods, might help to explain ER among Chinese international students and their personal stories in Ireland. 


\section{CONCLUSION}

The number of Chinese international students studying in Ireland is increasing, but there is limited research examining mental health among this population in Ireland. The current study investigated how ER influences perceived stress among Chinese international college students and compared them to their host country peers (i.e., Irish college students). The findings highlight the importance of considering nationality differences and cultural norms when examining ER and mental health among Chinese international college students in the new host country environment. More emphasis should be put on improving the understanding of the relationship between use of coping strategies and mental health among international students. HEIs could incorporate emotion coaching and stress management workshops into international students' induction programs. However, when stress supports are being provided by Irish HEIs to international students, consideration must be given to the kind of advice and supports provided, with an awareness of the cultural differences.

\section{REFERENCES}

Barrett, P. (2007). Structural equation modelling: Adjudging model fit. Personality and Individual Differences, 42(5), 815-824. https://doi.org/ 10.1016/j.paid.2006.09.018

Blalock, D. V., Kashdan, T. B., \& Farmer, A. S. (2016). Trait and daily emotion regulation in social anxiety disorder. Cognitive Therapy and Research, 40(3), 416-425. https://doi.org/10.1007/s10608-015-9739-8

Boyes, M. E., Hasking, P. A., \& Martin, G. (2016). Adverse life experience and psychological distress in adolescence: Moderating and mediating effects of emotion regulation and rumination. Stress and Health, 32(4), 402-410. https://doi.org/10.1002/smi.2635

Butler, E. A., \& Gross, J. J. (2009). Emotion and emotion regulation: Integrating individual and social levels of analysis. Emotion Review, 1(1), 86-87. https://doi.org/10.1177/1754073908099131

Butler, E. A., Lee, T. L., \& Gross, J. J. (2007). Emotion regulation and culture: Are the social consequences of emotion suppression culture-specific? Emotion, 7(1), 30. https://psycnet.apa.org/doi/10.1037/1528-3542.7.1.30

Campbell-Sills, L., Barlow, D. H., Brown, T. A., \& Hofmann, S. G. (2006). Acceptability and suppression of negative emotion in anxiety and mood disorders. Emotion, 6(4), 587. https://doi.org/10.1037/1528-3542.6.4.587

Carrico, A. W., Antoni, M. H., Weaver, K. E., Lechner, S. C., \& Schneiderman, N. (2005). Cognitive-Behavioural stress management with HIV-positive homosexual men: Mechanisms of sustained reductions in depressive symptoms. Chronic Illness, 1(3), 207-215. https://doi.org/10.1177/ 17423953050010030401

Chen, H., Akpanudo, U., \& Hasler, E. (2020). How do Chinese international students view seeking mental health services? Journal of International Students, 10(2), 286-305. https://doi.org/10.32674/jis.v10i2.765 
Cheng, M., Friesen, A., \& Adekola, O. (2019). Using emotion regulation to cope with challenges: A study of Chinese students in the United Kingdom. Cambridge Journal of Education, 49(2), 133-145. https://doi.org/10.1080/ 0305764X.2018.1472744

Chervonsky, E., \& Hunt, C. (2019). Emotion regulation, mental health, and social wellbeing in a young adolescent sample: A concurrent and longitudinal investigation. Emotion, 19(2), 270. https://doi.org/10.1037/emo0000432

Cho, J., \& Yu, H. (2015). Roles of university support for international students in the United States: Analysis of a systematic model of university identification, university support, and psychological well-being. Journal of Studies in International Education, 19(1), 11-27. https://doi.org/10.1177\% 2F1028315314533606

Clarke, M., Yang, L. H, \& Harmon, D. (2018). The internationalisation of Irish higher education. The Higher Education Authority.

Cohen, J. (1988). Statistical power analysis for the behavioral sciences. Routledge Academic.

Cohen, S., Kamarck, T., \& Mermelstein, R. (1983). A global measure of perceived stress. Journal of Health and Social Behavior, 24(4), 385-396. https://doi.org/10.2307/2136404

De Leersnyder, J., Mesquita, B., \& Kim, H.S. (2011). Where do my emotions belong? A study of immigrants' emotional acculturation. Personality and Social Psychology Bulletin, 37(4), 451-463. https://doi.org/10.1177/ 0146167211399103

English, T., John, O. P., Srivastava, S., \& Gross, J. J. (2012). Emotion regulation and peer-rated social functioning: A 4-year longitudinal study. Journal of Research in Personality, 46(6), 780-784. https://doi.org/10.1016/j.jrp. 2012.09.006

Flouri, E., \& Mavroveli, S. (2013). Adverse life events and emotional and behavioural problems in adolescence: The role of coping and emotion regulation. Stress and Health, 29(5), 360-368. https://doi.org/10.1002/ smi. 2478

Forbush, E., \& Foucault-Welles, B. (2016). Social media use and adaptation among Chinese students beginning to study in the United States. International Journal of Intercultural Relations, 50, 1-12. https://doi.org/10.1016/ j.ijintrel.2015.10.007

Ford, B. Q., \& Mauss, I. B. (2015). Culture and emotion regulation. Current Opinion in Psychology, 3(1), 1-5. https://doi.org/10.1016/j.copsyc. 2014.12.004

Frijda, N. H., \& Sundararajan, L. (2007). Emotion refinement: A theory inspired by Chinese poetics. Perspectives on Psychological Science, 2(3), 227-241. https://doi.org/10.1111/j.1745-6916.2007.00042.x

Goldin, P. R., McRae, K., Ramel, W., \& Gross, J. J. (2008). The neural bases of emotion regulation: Reappraisal and suppression of negative emotion. Biological Psychiatry, 63(6), 577-586. https://doi.org/10.1016/j.biopsych. 2007.05.031 
Gresham, D., \& Gullone, E. (2012). Emotion regulation strategy use in children and adolescents: The explanatory roles of personality and attachment. Personality and Individual Differences, 52(5), 616-621. https://doi.org/ 10.1016/j.paid.2011.12.016

Groarke, S., \& Durst, C. (2019). Attracting and retaining international higher education students. Economic and Social Research Institute.

Gross, J. J. (1998a). Antecedent-and response-focused emotion regulation: Divergent consequences for experience, expression, and physiology. Journal of Personality and Social Psychology, 74(1), 224. https://doi.org/ 10.1037/0022-3514.74.1.224

Gross, J. J. (1998b). The emerging field of emotion regulation: An integrative review. Review of General Psychology, 2(3), 271. https://doi.org/ 10.1037/1089-2680.2.3.271

Gross, J. J. (2001). Emotion regulation in adulthood: Timing is everything. Current Directions in Psychological Science, 10(6), 214-219. https://doi.org/10.1111/1467-8721.00152

Gross, J. J., \& John, O. P. (2003). Individual differences in two emotion regulation processes: Implications for affect, relationships, and well-being. Journal of Personality and Social Psychology, 85(2), 348-362. https://doi.org/ 10.1037/0022-3514.85.2.348

Gross, J. J. (2013). Emotion regulation: Taking stock and moving forward. Emotion, 13(3), 359. https://doi.org/10.1037/a0032135

Gross, J. J. (2015). Emotion regulation: Current status and future prospects. Psychological Inquiry, 26(1), 1-26. https://doi.org/10.1080/1047840X. 2014.940781

Han, S., Pistole, M. C., \& Caldwell, J. M. (2017). Acculturative stress, parental and professor attachment, and college adjustment in Asian international students. Journal of Multicultural Counseling and Development, 45(2), 111-126. https://doi.org/10.1002/jmcd.12068

Harrer, M., Adam, S. H., Fleischmann, R. J., Baumeister, H., Auerbach, R., Bruffaerts, R., Cuijpers, P., Kessler, R. C., Berking, M., Lehr, D., \& Ebert, D. D. (2018). Effectiveness of an internet-and app-based intervention for college students with elevated stress: Randomized controlled trial. Journal of Medical Internet Research, 20(4), e136. https://doi.org/ 10.2196/jmir.9293

Hofstede, G. (2001). Culture's consequences: Comparing values, behaviors, institutions and organizations across nations. SAGE.

Hu, T., Zhang, D., Wang, J., Mistry, R., Ran, G., \& Wang, X. (2014). Relation between emotion regulation and mental health: A meta-analysis review. Psychological Reports, 114(2), 341-362. https://doi.org/10.2466/03.20. PR0.114k22w4

Kwon, H., Yoon, K. L., Joormann, J., \& Kwon, J. H. (2013). Cultural and gender differences in emotion regulation: Relation to depression. Cognition \& Emotion, 27(5), 769-782. https://doi.org/10.1080/02699931.2013.792244 
Leong, P. (2015). Coming to America: Assessing the patterns of acculturation, friendship formation, and the academic experiences of international students at a U.S. college. Journal of International Students, 5(4), 459-474. https://www.ojed.org/index.php/jis/article/view/408

Matsumoto, D. (2006). Are cultural differences in emotion regulation mediated by personality traits. Journal of Cross-Cultural Psychology, 37(4), 421-437. https://doi.org/10.1177/0022022106288478

Matsumoto, D., Yoo, S. H., \& Nakagawa, S. (2008). Culture, emotion regulation, and adjustment. Journal of Personality and Social Psychology, 94(6), 925. https://doi.org/10.1037/0022-3514.94.6.925

Mauss, I. B., \& Butler, E. A. (2010). Cultural context moderates the relationship between emotion control values and cardiovascular challenge versus threat responses. Biological Psychology, 84(3), 521-530. https://doi.org/10.1016/ j.biopsycho.2009.09.010

Mauss, I. B., Butler, E. A., Roberts, N. A., \& Chu, A. (2010). Emotion control values and responding to an anger provocation in Asian-American and European-American individuals. Cognition and Emotion, 24(6), 1026-1043. https://doi.org/10.1080/02699930903122273

Mesquita, B. (2001). Emotions in collectivist and individualist contexts. Journal of Personality and Social Psychology, 80(1), 68. https://doi.org/10.1037/ 0022-3514.80.1.68

Minkov, M., Dutt, P., Schachner, M., Morales, O., Sanchez, C., Jandosova, J., Khassenbekov, Y., \& Mudd, B. (2017). A revision of Hofstede's individualism-collectivism dimension. Cross Cultural \& Strategic Management, 24(3), 386-404. https://doi.org/10.1108/CCSM-11-2016-0197

Moore, S. A., Zoellner, L. A., \& Mollenholt, N. (2008). Are expressive suppression and cognitive reappraisal associated with stress-related symptoms. Behaviour Research and Therapy, 46(9), 993-1000. https:// doi.org/10.1016/j.brat.2008.05.001

Myers, S. B., Sweeney, A. C., Popick, V., Wesley, K., Bordfeld, A., \& Fingerhut, R. (2012). Self-care practices and perceived stress levels among psychology graduate students. Training and Education in Professional Psychology, 6(1), 55. https://doi.org/10.1037/a0026534

Noy, C. (2008). Sampling knowledge: The hermeneutics of snowball sampling in qualitative research. International Journal of Social Research Methodology, 11(4), 327-344. https://doi.org/10.1080/13645570701401305

Oyserman, D., \& Lee, S. W. (2008). Does culture influence what and how we think? Effects of priming individualism and collectivism. Psychological Bulletin, 134(2), 311. https://doi.org/10.1037/0033-2909.134.2.311

Pakenham, K. I. (2005). Relations between coping and positive and negative outcomes in careers of persons with multiple sclerosis (MS). Journal of Clinical Psychology in Medical Settings, 12(1), 25-38. https://doi.org/ 10.1007/s10880-005-0910-3

Palinkas, L. A. (2014). Qualitative and mixed methods in mental health services and implementation research. Journal of Clinical Child \& Adolescent Psychology, 43(6), 851-861. https://doi.org/10.1080/15374416.2014.910791 
Potthoff, S., Garnefski, N., Miklósi, M., Ubbiali, A., Domínguez-Sánchez, F. J., Martins, E. C., Witthöft, M., \& Kraaij, V. (2016). Cognitive emotion regulation and psychopathology across cultures: A comparison between six European countries. Personality and Individual Differences, 98(1), 218-224. https://doi.org/10.1016/j.paid.2016.04.022

Rankin, J.A., Paisley, C. A., Mulla, M. M., \& Tomeny, T. S. (2018). Unmet social support needs among college students: Relations between social support discrepancy and depressive and anxiety symptoms. Journal of Counselling Psychology, 65(4), 474-489. doi: 10.1037/cou0000269.

Roberts, N. A., Levenson, R. W., \& Gross, J. J. (2008). Cardiovascular costs of emotion suppression cross ethnic lines. International Journal of Psychophysiology, 70(1), 82-87. https://doi.org/10.1016/j.ijpsycho.2008. 06.003

Sawir, E., Marginson, S., Deumert, A., Nyland, C., \& Ramia, G. (2008). Loneliness and international students: An Australian study. Journal of Studies in International Education, 12(2), 148-180. https://doi.org/10.1177/ 1028315307299699

Singelis, T. M., Triandis, H. C., Bhawuk, D. P., \& Gelfand, M. J. (1995). Horizontal and vertical dimensions of individualism and collectivism: A theoretical and measurement refinement. Cross-cultural Research, 29(3), 240-275. https://doi.org/10.1177/106939719502900302

Smith, R. A., \& Khawaja, N. G. (2011). A review of the acculturation experiences of international students. International Journal of Intercultural Relations, 35(6), 699-713. https://doi.org/10.1016/j.ijintrel.2011.08.004

Soto, J. A., Perez, C. R., Kim, Y. H., Lee, E. A., \& Minnick, M. R. (2011). Is expressive suppression always associated with poorer psychological functioning? A cross-cultural comparison between European Americans and Hong Kong Chinese. Emotion, 11(6), 1450. https://doi.org/10.1037/a0023340

Su, J. C., Lee, R. M., \& Oishi, S. (2013). The role of culture and self-construal in the link between expressive suppression and depressive symptoms. Journal of Cross-Cultural Psychology, 44(2), 316-331. https://doi.org/10.1177/ 0022022112443413

Su, J. C., Lee, R. M., Park, I. J., Soto, J. A., Chang, J., Zamboanga, B. L., Kim, S. Y., Ham, L. S., Dezutter, J., Hurley, E. A., Seol, K. O., \& Brown, E. (2015). Differential links between expressive suppression and well-being among Chinese and Mexican American college students. Asian American Journal of Psychology, 6(1), 15. https://doi.org/10.1037/a0036116

Sullivan, C., \& Kashubeck-West, S. (2015). The interplay of international students' acculturative stress, social support, and acculturation modes. Journal of International Students, 5(1), 1-11. https://www.ojed.org/ index.php/jis/article/view/438

Sun, M., \& Lau, A. S. (2018). Exploring cultural differences in expressive suppression and emotion recognition. Journal of Cross-Cultural Psychology, 49(4), 664-672. https://doi.org/10.1177/0022022118763749 
Thompson, R. A. (1994). Emotion regulation: A theme in search of definition. Monographs of the Society for Research in Child Development, 59(2-3), 2552. https://doi.org/10.2307/1166137

Troy, A. S., \& Mauss, I. B. (2011). Resilience in the face of stress: Emotion regulation as a protective factor. In S. Southwick, D. Charney, M. Friedman, \& B. Litz (Eds.), Resilience and mental health: Challenges across the life span (pp. 30-44). Cambridge University Press.

Troy, A. S., Wilhelm, F. H., Shallcross, A. J., \& Mauss, I. B. (2010). Seeing the silver lining: Cognitive reappraisal ability moderates the relationship between stress and depressive symptoms. Emotion, 10(6), 783. https://doi.org/ $10.1037 / \mathrm{a} 0020262$

Tsai, W., \& Lu, Q. (2018). Culture, emotion suppression and disclosure, and health. Social and Personality Psychology Compass, 12(3), 123. https://doi.org/10.1111/spc3.12373

Wei, M., Su, J. C., Carrera, S., Lin, S. P., \& Yi, F. (2013). Suppression and interpersonal harmony: A cross-cultural comparison between Chinese and European Americans. Journal of Counseling Psychology, 60(4), 625. https://doi.org/10.1037/a0033413

Westland, J. C. (2012). Erratum to "Lower bounds on sample size in structural equation modeling" [Electronic Commerce Research and Applications 9(6) (2010) 476-487]. Electronic Commerce Research and Applications, 11(4), 445.

Xiong, Y., \& Zhou, Y. (2018). Understanding East Asian graduate students' socio-cultural and psychological adjustment in a US midwestern university. Journal of International Students, 8(2), 769-794. https://doi.org/10.5281/ zenodo. 1250379

Yoo, S. H., Matsumoto, D., \& LeRoux, J. A. (2006). The influence of emotion recognition and emotion regulation on intercultural adjustment. International Journal of Intercultural Relations, 30(3), 345-363. https://doi.org/10.1016/ j.ijintrel.2005.08.006

Yuan, J., Liu, Y., Ding, N., \& Yang, J. (2014). The regulation of induced depression during a frustrating situation: Benefits of expressive suppression in Chinese individuals. PloS One, 9(5), Articlce 420. https://doi.org/10.1371/ journal.pone.0097420

Zahniser, E., \& Conley, C. S. (2018). Interactions of emotion regulation and perceived stress in predicting emerging adults' subsequent internalizing symptoms. Motivation and Emotion, 42(5), 1-11. https://doi.org/10.1007/ s11031-018-9696-0

YUNING SUN is a former student of the National College of Ireland and is a current $\mathrm{PhD}$ candidate in the Department of Psychology at the University of Limerick. His major research interests lie in the areas of mental health of international students and cultural differences in heroism. He can be reached via email at yuningsun9604@gmail.com. 
CONOR NOLAN, D.Psych.BAT, is a lecturer in psychology at the National College of Ireland. His major research interests are in the areas of behavioral psychology, mental health, and attitude research. He can be reached via email at conor.nolan@ncirl.ie. 